\title{
A Method for Studying the Enteroinsular Axis in Unanesthetized and Unrestrained Rats
}

\author{
Eiko HARA AND MASAYUKi SAITO \\ Department of Biochemistry, School of Medicine Ehime \\ University, Shigenobu, Ehime 791-02, Japan
}

\begin{abstract}
A method for studying the enteroinsular axis of rats under conditions comparable to those in experiments on human subjects was developed by chronically implanting an oral tube and two cardiac catheters into rats. These implantations were easy to perform and made it possible to give glucose and other stimulatory agents orally and/or intravenously and to take sequential blood samples without anesthetizing the rats or distrubing their movements. In this way, it was possible to confirm that oral loads of glucose and fat potentiate the insulin response induced by hyperglycemia.
\end{abstract}

It is well established that in human subjects the plasma insulin response to an oral load of glucose is much greater than that to an intravenous load of a comparable amount of glucose. This difference in the insulin response is thought to be due to the insulinotropic effect of certain gastrointestinal hormones that are released as a result of the oral glucose load. Recent results have suggested that gastric inhibitory polypeptide (GIP), which is secreted after oral but not intravenous glucose, is a leading candidate for mediating this enteroinsular axis (Brown et al., 1975; Anderson et al., 1978 ; Elahi et al., 1979).

The properties of the enteroinsular axis have been extensively studied in normal human subjects and patients with various types of diseases, but there have been few studies on this axis in laboratory animals under comparable conditions to those in humans. One reason for this is the difficulty of giving a definite amount of glucose orally during a certain fixed time and so

\footnotetext{
Received November 15, 1979.
}

strictly controlling the experimental conditions. Therefore, most studies have been made on anesthetized animals (Assal et al., 1970 ; Nijjar and Perry, 1970 ; Ebert et al., 1979; but see also Pederson et al., 1975), which are not physiologically comparable with unanesthetized human subjects. The present paper reports a simple method for studying the enteroinsular axis of unanesthetized rats without disturbing their movements. By this method it is possible to give an oral and/or intravenous load of glucose and/or other stimulatory agents and to take sequential blood samples.

\section{Methods}

\section{Surgical procedures}

Male Wistar rats weighing 300-350 g were anesthetized by intraperitoneal injection of sodium pentobarbital( $40 \mathrm{mg} / \mathrm{kg}$ body weight). An incision about $2 \mathrm{~cm}$ long was made in the skin of the head, and then an oral tube was implanted as follows. A small incision about $5 \mathrm{~mm}$ long was made in the skin of the right cheek, and a silicon tube $(0.5 \mathrm{~mm}$ i.d., $1 \mathrm{~mm}$ o.d., Ficon tube No. 00, Fuji Systems Co., Tokyo) was passed through the cheek wall into the 
oral cavity until its tip was $5 \mathrm{~mm}$ away from the wall of the cheek. The free end of the tube was attached to a metal stylet, which was passed under the skin to exit through the incision on the head. The end of the oral tube was connected to an Lneedle with a plastic wing (butterfly needle, Atom Co., Tokyo), which was fixed on the skull with a binding agent (Aron Alpha, Toa Gosei Kagaku Kogyo, Tokyo). A cardiac catheter for blood samplings was implanted by inserting a silicon tube $(0.5$ $\mathrm{mm}$ i.d., $1 \mathrm{~mm}$ o.d., Ficon tube No. 00) through a jugular vein into the auricle by the method of Steffens (1969). A cardiac catheter for glucose infusion was then implanted by inserting a polyethylene tube $(0.28$ mm i.d., 0.6 mm o.d., PE 10, Clay Adams, U.S.A.) through the jugular vein until its tip was about 5 $\mathrm{mm}$ down-stream of the tip of the sampling catheter (Steffens, 1969). The free ends of the cardiac catheters were both led subcutaneously to the incision in the head. The catheters were flushed and filled with saline solution containing heparin $(100 \mathrm{U} / \mathrm{ml})$ and then their free ends were closed with small metal-wire plugs. The ends of the cardiac catheters were secured to the butterfly needle with silk sutures, and then the incisions were closed. Postoperatively, the rats were housed individually.

\section{Experimental procedure}

Three days after the operation, the rats were starved overnight, and then their plasma glucose and insulin responses to oral and/or intravenous loads of glucose and fat were examined without the use of an anesthetic. For the tests, one end of a $50 \mathrm{~cm}$ extension tube $(0.58 \mathrm{~mm}$ i.d., $0.92 \mathrm{~mm}$ o.d., PE 50) was connected to the butterfly needle on the head, and the other end to a pump (Fig. 1). The metal-wire plug was removed from the end of the sampling catheter, and free blood flow in the catheter was established by flushing the tube with saline solution containing heparin. Then $0.3 \mathrm{ml}$ of saline solution containing heparin $(100 \mathrm{U} / \mathrm{m} l)$ was injected through the catheter. The catheter was then joined to a pump by a $50 \mathrm{~cm}$ extension tube $(0.58 \mathrm{~mm}$ i.d., $0.97 \mathrm{~mm}$ o.d., PE 50) filled with the saline solution. The infusion catheter was also connected to a pump by an extension tube in the same way. When the rats became accustomed to these fittings (usually within 10-20 $\mathrm{min}$ ), blood sampling was started. Blood was taken continuously at a constant rate of $20 \mu \mathrm{l} /$ min, diluted 10 -fold with saline solution, and collected every $2 \mathrm{~min}$ in tubes in a fraction collector. Several minues after the start of blood sampling, glucose solution $(1 \mathrm{~g} / 3 \mathrm{ml})$ and/or soy-bean oil $(2.5$ $\mathrm{g} / 3 \mathrm{ml}$ ) was injected into the mouth through the oral tube at a constant rate of $1 \mathrm{ml} / \mathrm{min}$ for $3 \mathrm{~min}$. For intravenous administration, $10 \%$ glucose solution was infused through the infusion catheter at a constant rate of $50 \mu \mathrm{l} / \mathrm{min}$, and blood sampling was continued for a further $20-30 \mathrm{~min}$.

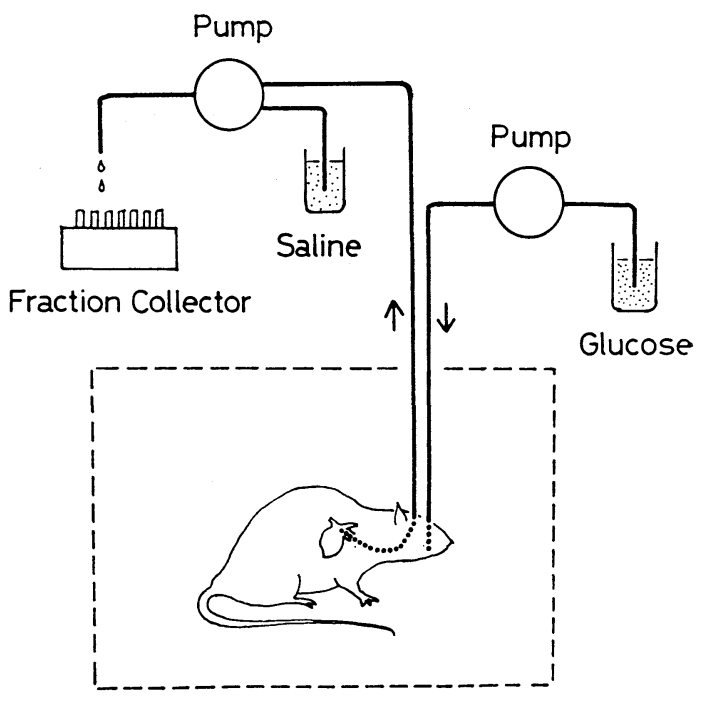

Fig. 1. Diagram of the system used in examining the plasma glucose and insulin responses to an oral and/or intravenous load of glucose and/or other stimulatory agents in unanesthetized, unrestrained rats.

\section{Analytical procedures}

The blood samples were cooled and centrifuged at $5,000 \times g$ for $10 \mathrm{~min}$. Aliquots of the resulting supernatants were assayed for glucose using a specific glucose oxidase methed (Automatic A-test, Boehringer $\mathrm{GmbH}$, Germany) and for immunoreactive insulin by the double antibody dethod (Insulin RIA kit, Dainabot Co., Tokyo). Statistical significance of differences between values was determined by Student's $t$ test.

\section{Results and Discussion}

In the present work, to study the plasma glucose and insulin responses of rats to various loads of stimulatory agents under conditions comparable to those in human subjects, we implanted the animals with an oral tube for oral loading and two cardiac catheters, one for intravenous loading and the other for blood sampling. Several methods have been developed for studying glucose tolerance and insulin secretion in unanethetized rats without disturbing their movements (Steffens, 1969 ; Louis-Sylvestre, 
1976; Shah et al., 1977). In most earlier studies, blood samples were taken through an indwelling cardiac catheter, as in the present study, but various methods have been used for glucose loading. When glucose was given through the gastrointestinal tract, it was given through an indwelling gastric tube (Louis-Sylvestre, 1976) or by allowing the rats to eat voluntarily (Steffens, 1969). In the former method, it is possible to load a fixed amount of glucose, but the procedure differs from that of the oral glucose tolerance test in human subjects in that the sugar does not enter the oral cavity. In the latter method, it is difficult to load a fixed amount of glucose during a fixed time. To overcome these difficulties, in the present study, we implanted a silicon tube into the oral cavity. When a glucose solution or soy-bean oil was injected into the mouth through the oral tube, the rats drank all the solution voluntarily, and none ran out of their mouth. Thus, it was possible to give a definite dose in a fixed time.

To load glucose intravenously, we implanted a second catheter in addition to the sampling catheter into the jugular vein, according to the two catheter method described by Steffens (1969). In order to prevent contamination of the blood sample with the infusion fluid, it may be best to implant the infusion catheter into a different position from the sampling catheter; for example, one into the jugular vein and the other into the aorta (Shah et al., 1977). However, these procedures are complicated and time-consuming. The two catheter method used in the present study is very easy, and there is practically no contamination of the blood sample with the infusion fluid if the end of the infusion catheter is a few millimeters down-stream from the tip of the sampling catheter.

The surgical technique required is therefore rather simple and easy, and all procedures can be completed within $30 \mathrm{~min}$. Postoperatively, the rats regained weight and their food intake returned to normal within two or three days. In more than 80 per cent of the animals, the cardiac catheters remained functional for ten days when the catheters were flushed once every day, and the oral tube for three weeks.

Fig. 2 shows the plasma glucose and insulin responses to oral and intravenous loads of glucose in unanesthetized rats. The basal levels of plasma glucose and insulin were about $85 \mathrm{mg} / \mathrm{d} l$ and $27 \mu \mathrm{U} / \mathrm{m} l$, respectively. When $1 \mathrm{~g}$ of glucose was given orally the levels of glucose and insulin rose rapidly to rather steady levels of about $125 \mathrm{mg} / \mathrm{d} l$ and $130 \mu \mathrm{U} / \mathrm{m} l$, respectively, within 15 min. When $10 \%$ glucose solution was infused intravenously at a constant rate of $50 \mu \mathrm{l} / \mathrm{min}$ the plasma glucose curve was similar to that after an oral glucose load, but the insulin level rose much more slowly and did not reach such a high level. Thus, the plasma insulin response to an oral glucose load was much greater than that to an intravenous glucose load, although the degree of hyperglycemia was similar. These results agree well with those in human subjects, and confirm the presence of an enteroinsular axis in rais (Assal et al., 1970 ; Ebert et al., 1979).

If the enteroinsular axis is mediated by GIP, an oral load of fat should potentiate the insulin response induced by hyperglycemia, because both fat and glucose stimulate GIP release in human subjects (Brown et al., 1975; Falko et al., 1975). To test this in unanesthetized rats, plasma glucose and insulin responses to an oral load of fat were examined with or without intravenous glucose. As shown in Fig. 3, when $3 \mathrm{~m} l$ of soy-bean oil was given orally, the levels of plasma glucose and insulin did not change significantly. Thus, the oral load of fat does not elicit insulin release under euglycemic conditions. However, when the oil was given orally during intravenous infusion of glucose, the insulin response was much greater than that after 

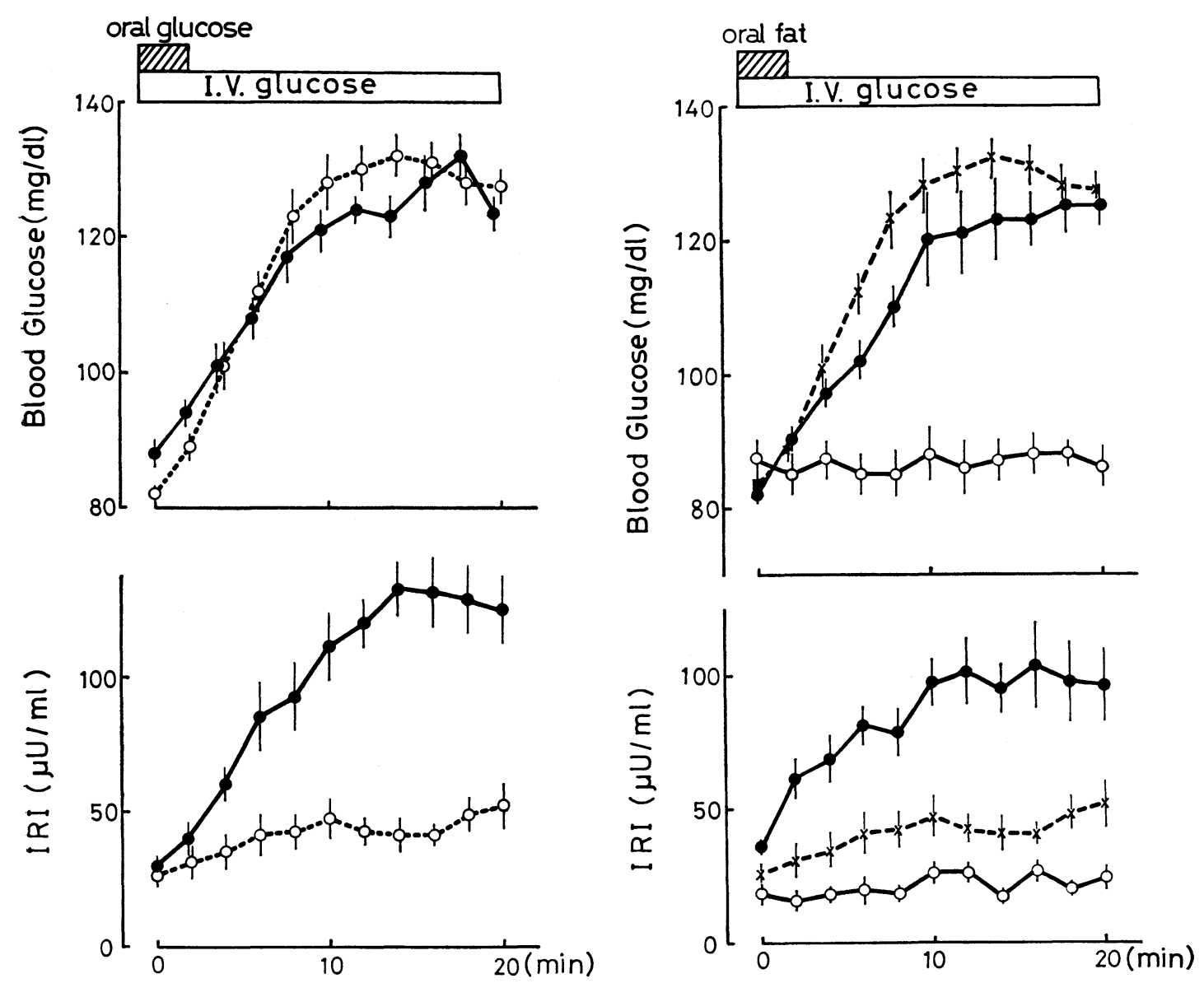

Fig. 2. Plasma glucose and insulin responses to glucose loads. Glucose was given orally by injecting $3 \mathrm{ml}$ of a solution $(1 \mathrm{~g} / 3 \mathrm{ml})$ in $3 \mathrm{~min}$ through the oral tube (-@), or intravenously by infusing a solution $(0.1 \mathrm{~g} / \mathrm{ml})$ through the cardiac catheter at a constant rate of $50 \mu \mathrm{l} / \mathrm{min}$ $(\circ \cdots \cdots \circ)$. The insulin levels after the oral glucose load were significantly higher than those after the intravenous glucose load at $4 \mathrm{~min}(\mathrm{P}<0.02)$ and later times $(\mathbf{P}<0.001)$. Values are means \pm $\mathrm{SE}$ of those in 8 rats.

intravenous infusion of glucose alone. Thus, an oral load of fat potentiates insulin release induced by hyperglycemia. These results are consistent with the finding in human subjects that endogenous GIP does not augment insulin release unless the blood

Fig. 3. Plasma glucose and insulin responses to an oral load of fat with or without intravenous glucose. Three $\mathrm{m} l$ of soy-bean oil was given orally with (-) or without ( glucose as for Fig. 2. The broken lines indicate the responses to the intravenous glucose load without an oral load of soy-bean oil. The insulin levels after the oral oil load with intravenous glucose were significantly higher than those after intravenous glucose alone at 2 and $4 \mathrm{~min}(\mathrm{P}<0.02)$ and later times $(P>0.005)$. Values are means \pm $\mathrm{SE}$ of those in 6 rats.

glucose level is raised to at least $20 \mathrm{mg} / \mathrm{d} l$ more than the normal level (Andersen et al., 1978).

As mentioned above, the present method is useful in studies on the plasma glucose and insulin response to various stimulatory 
agents given orally and/or intravenously in small laboratory animals under conditions comparable with those of the oral and/or intravenous glucose tolerance test in human subjects. The method seems especially useful for studying the role of the gastrointestinal tract in pancreatic secretion, because it is possible to control the blood glucose level independently from the oral lord. Furthermore, since the catheters and and oral tube remain functional for at least ten days, several exeriments can be performed on each animal.

\section{Acknowledgement}

We are greatly indebted to Prof. Masami Suda for his suggestions, and also to Miss Hiroko Noma for her technical assistance. This study was supported, in part, by a grant for scientific research from the Ministry of Education, Science and Culture of Japan.

\section{References}

Andersen, D. K., D. Elahi, J. C. Brown, J. D. Tobin and R. Andres (1978). J. Clin. Invest. 62, 152.

Assal, J. M., R. Levrat and W. Stauffacher (1970). Diabetologia 6, 35.

Brown, J. C., J. R. Dryburgh, S. A. Ross and J. Dupre (1975). Rec. Prog. Horm. Res. 31, 487.

Ebert, R., K. Illmer and W. Creutzfeldt (1979). Gastroenterology 76, 515.

Ehahi, D., D. K. Andersen, J. C. Brown, E. T. Debas, R. J. Hershcopf, G. S. Raizes, J. D. Tobin and R. Andres (1979). Am. J. Physiol. 237, E185.

Falko, J. M., S. E. Crockett, S. Cataland and E. L. Mazzaferri (1975). J. Clin. Endocrinol. Metab. 41, 260.

Louis-Sylvestre, J. (1976). Am. J. Physiol. 230, 56.

Nijjar, M. S. and W. F. Perry (1970). Diabetes 19, 155.

Pederson, R. A., H. E. Schubert and J. C. Brown (1975). Ibid. 24, 1050.

Shah, J. H., N. Wongsurawat, P. P. Aran, G. S. Motto and E. N. Bowser (1977). Ibid. 26, 1.

Steffens, A. B. (1969). Physiol. Behav. 4. 833. 